\title{
Vertical water entry of projectiles with surface seal
}

\author{
Yufei Wang ${ }^{\mathrm{a}, \mathrm{b}}$, Bingsheng Ye ${ }^{\mathrm{a}, \mathrm{b}}$, Zhiying Wang ${ }^{\mathrm{a}, \mathrm{b}}$, Jian Huang ${ }^{\mathrm{b}}$, Yiwei Wang ${ }^{\mathrm{a}, \mathrm{b}, *}$, \\ Chenguang Huang ${ }^{\text {a,b }}$
}

${ }^{a}$ Key Laboratory for Mechanics in Fluid Solid Coupling Systems, Institute of Mechanics, Chinese Academy of Sciences, Beijing, 100190, China

${ }^{\mathrm{b}}$ School of Engineering Science, University of Chinese Academy of Sciences, Beijing, 100049, China

\section{A B S T R A C T}

The water entry of a three-dimensional slender projectile with a constant vertical velocity is investigated numerically, and experiments are done to validate numerical results. We limit the scope of this work to the cavity forming stage at which surface seals. Typical experiments are carried out in a launching system. A pressure-based compressible multiphase solver combined with the Kunz cavitation model is developed within the OpenFOAM ${ }^{\circledR}$ platform. The numerical and experiment results show a good agreement. The time-dependent change regularity of the drag coefficient, pressure distribution, air-entrained cavity evolution, and flow characteristics inside the cavity are elaborated. Their relationships with the key parameter Froude number are also discussed. Results indicate that the drag coefficient after the impact phase is approximately equal to that in supercavitation case, and it is generally of the Froude number. Cavity shapes are generally independent of Froude number in the early stage of water entry. However, discrepancies in profiles gradually appear at a later time. For the airflow characteristics, circumferential vortex rings are found near the free surface in the early stage of water entry. The pressure inside the cavity decreases with time because of the rapid growth in cavity volume, which the entrained air cannot compensate for.

\section{Introduction}

Water entry refers to the process in which solid bodies pass through free surfaces from air into liquids. This phenomenon is common in nature, e.g., seabirds diving into water to catch fish (Chang et al. 2016), animals walking on water (Hu et al. 2003; Hsieh and Lauder, 2004), and stones ricocheting when thrown in water (Clanet et al. 2004; Nagahiro and Hayakawa, 2005). Water entry is also essential for a wide range of engineering applications, such as slamming loads caused by ship-wave interaction (Wang and Soares, 2016), coastal protection facilities (Sruthi and Sriram, 2017), and water landing and water impact of spacecraft. The literature about water entry for aerospace structures was collected and presented by Seddon and Moatamedi (2006) sufficiently. Relevant research about military applications, e.g., air to water missiles (May, 1953; May, 1975), supercavitating vehicles during phase transition (Kulkarni and Pratap, 2000), and water bouncing bomb (Johnson, 1998) have also been identified.

Water entry has been a hotspot in the fluid mechanics field over the past decade. Forces and cavity or splash morphological evolution are important considerations and are widely investigated in mechanical studies (Vincent et al., 2018). Truscott et al. (2012) reported that the forces in cavity-forming and noncavity-forming cases and that such forces are controlled by the unsteady pressure required to expand and shrink air cavities. The influence of different head shapes or angles of axisymmetric objects on drag coefficient and pressure distribution have been discussed in detail, and results indicated that flat cylinders experienced the largest impulsive forces during water entry (e.g., Niazmand et al. 2017; Bodily et al. 2014). Vincent et al. (2018) measured the forces acting on wedges with different angles for Froude ( Fr) numbers ranging from 1.7 to 5 . Large wedge angles underwent a peak force before submersion, whereas small wedge angles were associated with smoothly increasing drag, especially when they are equal to $90^{\circ}$. The drag coefficient was independent of the $\mathrm{Fr}$ number at the cavity forming stage. $\mathrm{Xu}$ et al. (2008) investigated oblique hydrodynamic entry numerically and revealed that wave elevation, pressure distribution, and force at different inclination angles were distinct from those in vertical entry cases. Panciroli et al. (2015) experimentally studied the fluid-structure interaction during water entry in flexible cylinders and found that deformation takes place in the direction of hydrodynamic load and that vibration amplitude increases with entry velocity. When spin was considered, the pressure of the downstream side was lower than that of upstream spinning side; hence, a lateral force was generated, and pressure fluctuations became evident in the upstream side (Kiara et al. 2017).

Water entry dynamics have been continuously studied in terms of cavity profiles and splash shapes since Worthington (1908) recorded the water entry process through a photographic study. The water entry

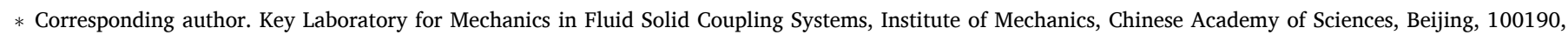
China.

E-mail address: wangyw@imech.ac.cn (Y. Wang).
} 


\begin{tabular}{|ll|}
\hline \multicolumn{2}{|l|}{ Nomenclature } \\
\multicolumn{2}{|l|}{ Latin letters } \\
$a_{0} \quad$ projectile radius $(\mathrm{m})$ \\
$a$ & cavity radius $(\mathrm{m})$ \\
$A$ & section area of projectile $\left(\mathrm{m}^{2}\right)$ \\
$C d$ & drag coefficient \\
$C p$ & pressure coefficient \\
$D$ & projectile diameter $(\mathrm{m})$ \\
$F d$ & average drag force $(\mathrm{N})$ \\
$L$ & projectile length $(\mathrm{m})$ \\
$P g$ & pressure drop across the air-water interface $(\mathrm{Pa})$ \\
$p_{v}$ & saturated vapor pressure $(\mathrm{Pa})$ \\
$t^{*}$ & non-dimensional time \\
$v_{0}$ & initial water entry velocity $(\mathrm{m} / \mathrm{s})$ \\
Greek letters \\
$\alpha_{l}$ & water volume fraction \\
$\alpha_{v}$ & vapor volume fraction \\
$\alpha_{a}$ & air volume fraction \\
$\rho_{s}$ & projectile density $\left(\mathrm{kg} / \mathrm{m}^{3}\right)$ \\
$\rho_{l}$ & water density $\left(\mathrm{kg} / \mathrm{m}^{3}\right)$ \\
$\rho_{a}$ & air density $\left(\mathrm{kg} / \mathrm{m}^{3}\right)$ \\
$\sigma$ & water surface tension $\left(\mathrm{kg} / \mathrm{s}^{2}\right)$ \\
$\mu$ & dynamic viscosity of water $\left(\mathrm{N} \cdot \mathrm{s} / \mathrm{m}^{2}\right)$ \\
\hline
\end{tabular}

problem can be governed by a series of dimensionless parameter, and different phenomena show varied scopes. Aristoff and Bush (2009) revealed four possible cavity types of air chambers after hydrophobic solid bodies penetrating into water: surface seal, deep seal, shallow seal, and quasi-static seal. Surface and deep seals are the most general forms in previous literatures. In deep seal case, cavity closure occurs approximately halfway between the pool surface and the object. Surface seal is characterized by cavity pinch-off at the free surface due to splash move inward. Surveys conducted by Lee et al. (1997) indicated that deep seal precedes surface seal when $\sqrt{20}<F r<\sqrt{70}$ and that surface seal precedes deep seal when $\mathrm{Fr}>\sqrt{150}$. However, existing research tended to focus on deep seal cases rather than surface seal cases, and several flow characteristics and regulations in surface closure case remained unclear (Truscott et al., 2014). In addition to the Fr number, a series of dimensionless parameters can also affect the properties of water entry; including the Weber (We) number, Bond number, Reynolds number, mass ratio, and spin ratio. The Weber number and Bond number are related to surface tension, which are known to influence cavity types and splash development. Recently, the fine and detailed descriptions of cavity features have become an important concern. Louf et al. (2018) studied cavity dynamics in the long term and measured the amplitude and wavelength of ripples that formed after cavity pinch-off. Tan et al. (2016) investigated the problem of a sphere falling into a stratified two-layer oil-water medium, measured the unstable ripple-like structures induced in the cavity boundary, and compared homogeneous systems and two-layer systems in terms of cavity formation. Marston et al. (2016) reported that atmospheric pressure significantly influences the timescale of splash crown closure and that a splash dome takes a long time to form when ambient pressure decreases.

Unfortunately, limited research has explored cavity pressure. Abelson (1970) recorded cavity pressure data using underwater probes and found that the faster the initial velocity was, the greater the decrease in pressure would be; moreover, results indicated that cavity pressure was governed by the isentropic flow-volume relation after cavity closure and that high-pressure pulses could be observed when a cavity collapsed. These rough experiments were carried out nearly 50 years ago, and the conclusions need to be revalidated. Only when we sufficiently consider atmospheric pressure and combine it with hydraulic pressure can we establish a complete model to describe cavity evolutions. Insights into the internal flow of cavities are fundamental.

Many researchers have studied these issues using theoretical and experimental methods. However, numerical methods have been increasingly used since von Kármán (1929) first proposed the theoretical method for solving the impact loads of 2D wedges. Wagner (1932) introduced potential flow theory and free surface conditions on the basis of von Kármán's theory and precision was improved in the calculation results. Many scholars have used various theories to calculate slamming load when an object enters water, and these theories include those by Dobrovol'skaya (1969), Howison et al. (1991), Mei et al. (1999), and Korobkin and Scolan (2006). However, the theories were based on the ideal hypothesis that liquid was incompressible, inviscid, and irrotational. As for cavity and splash dynamics, Lee et al. (1997) established formulas for predicting the radius of cavitation from an energy conservation perspective. Duclaux et al. (2007) combined the traditional Besant-Rayleigh approach with potential flow and derived a solution to describe the evolution in time of the cavity profile in terms of water entry problems for cylinders and spheres. Aristoff and Bush (2009) presented a theoretical model that considers underwater cavity dynamics and applied it to the theoretical analysis of splash curtain evolution. Experimental and numerical methods are widely adopted. Relevant experiments are mostly conducted in water tanks by employing accurate measuring methods, such as high-speed cameras, particle image velocimeters (e.g., Mansoor et al., 2014; Shams et al., 2015; Wang et al., 2017; Xu et al., 2018; Speirs et al., 2019; Kim and Park, 2019), and force sensors (e.g., Chang et al., 2016; Vincent et al., 2018; Louf et al., 2018). However, in most studies, the object launch systems were simply constructed so that the initial entry velocity was less than $10 \mathrm{~m} / \mathrm{s}$ generally. With the advancement of computing, numerical simulations have become another important approach to solve water entry problems. Water entry can form a multiphase flow with large deformation of the free surface. Hence, selecting the method to define and track free surfaces is particularly critical. The boundary element method (BEM) in the framework of potential flow has been applied to the water entry of 2D objects by many researchers, such as Zhao and Faltinsen (1993), Battistin and Iafrati (2003), and Sun and Faltinsen (2006). Various schemes, e.g., the coupled Eulerian-Lagrangian technique (Erfanian et al., 2015), meshless Lagrangian smoothed particle hydrodynamics (Kiara et al., 2017; Gong et al., 2019), level set (Calderer et al., 2014), immersed boundary (Mcintyre et al., 2011), and lattice Boltzmann (Zarghami et al., 2014), are all useful approaches to simulate cavity profiles and splash scatter.

Almost all the aforementioned studies focused on deep seal cases rather than surface seal cases. Water entry occurs at a low speed, and because of limitations in research methods, the forces and cavity dynamics for surface seal cases are still unclear. In the current work, a 3D slender conical head projectile is adopted as the research object to investigate vertical water entry problems under surface seal cases via experiments and numerical simulation. Table 1 shows the relevant nondimensional parameters and their definitions. The influence of $\mathrm{Fr}$ number is discussed to fill the gap because only a few studies considered surface seal cases with a high Fr number. Temporal evolutions of cavities with a range of $F r$ number are examined. The detailed cavity pressure distribution is presented and discussed. A numerical model for solving Navier-Stokes equations is employed on the basis of large eddy simulation (LES) and the volume of fluid (VOF) method. Our previous works show that this model can simulate complex multiphase flow characteristics and depict sharp interfaces (Ye et al. 2019) and that its results are in good agreement with experimental outcomes. A pressure-based compressible multiphase solver with cavitation phase change is also developed within the OpenFOAM ${ }^{\circledR}$ source code. Evidence shows that air and water compressibility cannot be neglected, especially when $\mathrm{Fr}$ is relatively high (Hicks et al. 2012; Elhimer et al. 2017). A set of new experimental equipment with a high-speed camera and a 
Table 1

Relevant nondimensional parameters that affect the water entry problem, their definitions, and characteristic values in the present study. A projectile penetrates into water with the initial velocity of $V_{0} \in[30,50]$, given the water surface tension of $\sigma=7.34 \times 10^{-2} \mathrm{~kg} \mathrm{~s}^{-2}$.

\begin{tabular}{llll}
\hline Nondimensional parameters & Abbreviation & Definition & Magnitude \\
\hline Weber number & We & $\frac{\rho_{l} v_{0}^{2} L}{\sigma}$ & $10^{6}-10^{7}$ \\
Bond number & Bo & $\frac{\rho_{l} g L^{2}}{\sigma}$ & $10^{3}$ \\
Froude number & $F r$ & $\frac{v_{0}}{\sqrt{g L}}$ & $10^{2}$ \\
Reynolds number & $R e$ & $\frac{\rho v_{0} L}{\mu}$ & $10^{6}$ \\
Mass ratio (solid-water) & $m *$ & $\frac{\rho_{s}}{\rho}$ & 10 \\
Mass ratio (air-water) & $m$ & $\frac{\rho_{a}}{\rho}$ & $10^{-3}$ \\
Cavitation number & $Q$ & $\frac{p-p_{v}}{0.5 \rho v_{0}^{2}}$ & $10^{-1}$ \\
\hline
\end{tabular}

pressure-driven launch system is designed and established to guarantee the entry velocity. The simulation and experiments are compared to validate the simulation results.

\section{Experimental setup}

The launch system used for water entry experiments consists of four sections, namely, a movable pressure-driven launcher with a gun barrel, a sealed water tank, a pneumatic regulating device, and a launching trigger. The upper part of the sealed water tank is equipped with a quadrangular bracket, on which a horizontal guide rail and mobile launcher are fixed. High-pressure gas is applied as the power source and controlled with a solenoid valve. The projectile specimen is shot out of the barrel at a speed of up to $60 \mathrm{~m} / \mathrm{s}$. Projectile velocity is defined as the temporal derivatives of the measured position data (Truscott et al., 2012), and we use smoothing spline method to fit the position-time curve in order to minimize the error to measured data. The launch angle is made adjustable.

The whole process of water entry is recorded by a high-speed camera with an exposure frequency of 20,000 frames per second. The specimen used in the experiment is an aluminum conical cylinder with a length of $90 \mathrm{~mm}$ and a diameter of $9 \mathrm{~mm}$. Fig. 1 shows the schematic of our experimental equipment.

\section{Numerical models}

\subsection{Governing equations}

The VOF method is adopted to capture free surface motion; here, $\alpha_{l} \alpha_{v^{\prime}} \alpha_{a}$ are the water, vapor, and air volume fractions, respectively, and must satisfy

$\alpha_{l}+\alpha_{v}+\alpha_{a}=1$.

The phase change between liquid water and vapor is introduced by the TEM-derived cavitation model. In terms of compressible fluid, the mass conservation equation of each phase can be written as

$$
\begin{aligned}
& \frac{\partial \alpha_{l}}{\partial t}+\nabla \cdot\left(\alpha_{l} \mathbf{u}\right)=-\frac{\alpha_{l}}{\rho_{l}} \frac{D \rho_{l}}{D t}+\frac{\dot{m}}{\rho_{l}} \\
& \frac{\partial \alpha_{v}}{\partial t}+\nabla \cdot\left(\alpha_{v} \mathbf{u}\right)=-\frac{\alpha_{v}}{\rho_{v}} \frac{D \rho_{v}}{D t}-\frac{\dot{m}}{\rho_{v}}, \\
& \frac{\partial \alpha_{a}}{\partial t}+\nabla \cdot\left(\alpha_{a} \mathbf{u}\right)=-\frac{\alpha_{a}}{\rho_{a}} \frac{D \rho_{a}}{D t}
\end{aligned}
$$

where $\dot{m}$ represents the mass exchange within each volume phase and $\rho_{l}, \rho_{v^{\prime}} \rho_{a}$ are the water, vapor, and air density, respectively. When the (a)

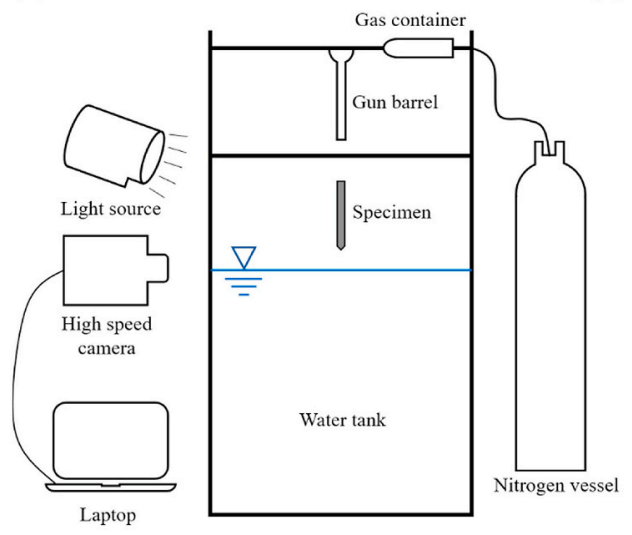

(b)

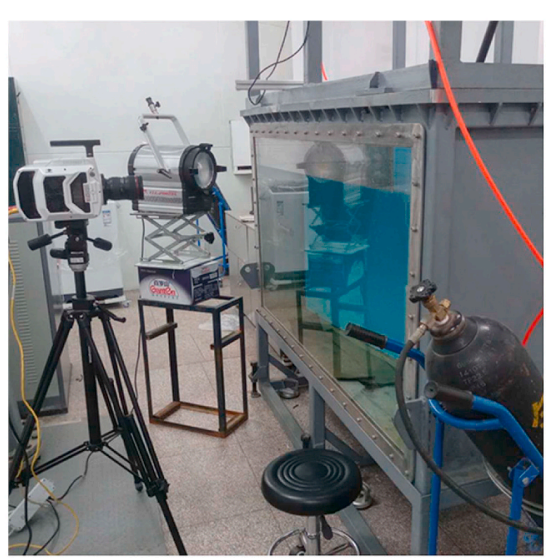

Fig. 1. Schematic of experimental launching system with glass water tank and high-speed camera. (a) Projectile specimen passing through the gun barrel before impacting the water surface. The gun barrel is perpendicular to the horizontal plane. An inert gas nitrogen is admitted into the gas container from a vessel. The internal pressure of the gas container determines the initial velocity of the specimen. (b) Actual experimental scene. (c) High-speed launching system. The initial angle can be adjusted, and the gun barrel is fixed vertically in our experiments.

(c)

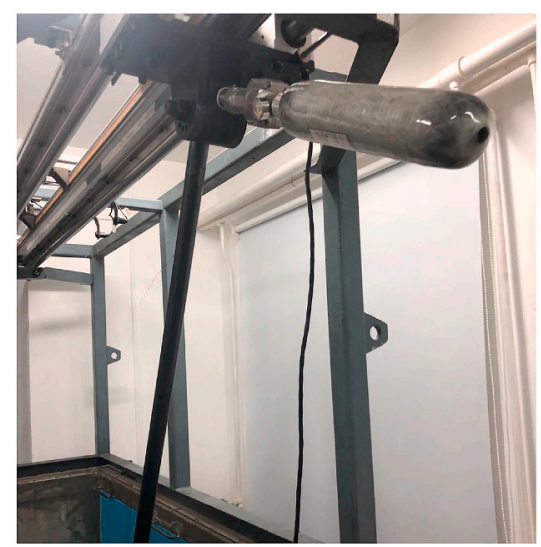


local pressure is less than the saturated vapor pressure $P_{\text {sat }}$, the liquid evaporates into vapor. When the local pressure is higher than the saturated vapor pressure, the water vapor condenses into liquid. The evaporation and condensation rates can be described by the Kunz model (Kunz et al. 2000).

$\dot{m}^{+}=\frac{C_{v} \rho_{v} \alpha_{l} \max \left(P_{s a t}-p, 0\right)}{0.5 \rho_{l} U_{\infty}^{2} t_{\infty}}$

$\dot{m}^{-}=\frac{C_{c} \rho_{v} \alpha_{v}^{2}\left(1-\alpha_{v}\right)}{t_{\infty}}$

where $\dot{m}^{+}$and $\dot{m}^{-}$represent the mass transfer rate of evaporation and condensation, The empirical constants for evaporation and condensation are denoted by $\mathrm{C}_{\mathrm{v}}$ and $\mathrm{C}_{\mathrm{c}}$, and the constants are set as: $C_{v}=200$ and $C_{c}=100$.

The momentum equation for the mixture is as follows:

$\frac{\partial \rho \mathbf{u}}{\partial t}+\nabla \cdot(\rho \mathbf{u u})=-\nabla p+\nabla \cdot\left[\mu\left(\nabla \mathbf{u}+(\nabla \mathbf{u})^{T}-\frac{2}{3}(\nabla \cdot \mathbf{u}) \mathbf{I}\right)\right]+\rho \mathbf{g}+\sigma \kappa \nabla \alpha_{1}$,

mixture viscosity $\mu$ and mixture density $\rho$ are defined as follows:

$\rho=\rho_{l} \alpha_{l}+\rho_{v} \alpha_{v}$

$\mu=\mu_{l} \alpha_{l}+\mu_{v} \alpha_{v}$

while $\mu, \sigma, \kappa$ respectively represents dynamic viscosity, surface tension coefficient and curvature.

The energy equation for the mixture is:

$\frac{\partial}{\partial t}[\rho(K+\varepsilon)]+\nabla \cdot[\rho \mathbf{u}(K+\varepsilon)]=\rho \mathbf{g} \cdot \mathbf{u}-\nabla \cdot q-\nabla \cdot(p \mathbf{u})+\nabla \cdot(\mathbf{u} \cdot \tau)$.

where $\varepsilon$ and $q$ denote the specific internal energy and heat flux density, respectively. Kinetic energy is defined as $K=1 / 2|u|^{2}$. Apart from disregarding the effect of shear stress, the following relations can be substituted into the energy equation:

$\varepsilon=C_{p} T$

$\mathbf{q}=-\alpha_{d} C_{p} \nabla T$.

An equation for temperature $T$ can be derived as follows:

$\frac{\partial}{\partial t}\left(\rho C_{p} T\right)+\nabla \cdot\left(\rho C_{p} T \mathbf{u}\right)+\frac{\partial}{\partial t}(\rho K)+\nabla \cdot(\rho K \mathbf{u})-\nabla \cdot\left(\alpha_{d} \nabla T\right)=-\nabla \cdot(p \mathbf{u})$,

where $C_{p}$ denotes the specific heat capacity of the mixture and $\alpha_{d}$ is the diffusion coefficient. Furthermore, the finite volume method is used to discretize the governing equations. Note that the new solver was developed by interPhaseChangeFoam framework in OpenFOAM. Our previous work (Ye et al., 2019) demonstrates the new solver is reliable in predicting compressible and cavitation flow.

\subsection{Turbulence modeling}

Large eddy simulations is considered as an important means to simulate turbulence flow. The LES approach divides vortices into large scales and small scales using a filtering operation in a selected field. The large scales are resolved directly from the instantaneous Navier-Stokes equation while the small scales are represented by subgrid scale (SGS) models. LES performs better than the traditional Reynolds-averaged Navier-Stokes approach in capturing details of unsteady flow structures and predicting eddies in turbulence.

In LES, the filtered large-scale quantity is denoted by a bar, and implicit filtering is employed in OpenFOAM $®$. The Favre filtering operation is applied to eliminate subfilter terms.

$\tilde{f}=\overline{\rho f} / \bar{\rho}$

The Favre-filtered momentum and energy equations can be written $\frac{\partial}{\partial t}(\bar{\rho} \tilde{u})+\nabla \cdot(\bar{\rho} \tilde{u} \tilde{u})=-\nabla \bar{p}+\nabla \cdot \bar{\tau}+\sigma k \nabla \overline{\alpha_{1}}+\nabla \cdot \tau_{S G S}$,

$$
\begin{gathered}
\frac{\partial}{\partial t}\left(\bar{\rho} C_{v} \tilde{T}\right)+\nabla \cdot\left(\bar{\rho} C_{v} \tilde{T} \tilde{u}\right)+\frac{\partial}{\partial t}\left(\bar{\rho} \frac{\tilde{u} \cdot \tilde{u}}{2}\right)+\nabla \cdot\left(\bar{\rho} \frac{\tilde{u} \cdot \tilde{u}}{2} \tilde{u}\right) \\
=\nabla \cdot\left(\alpha^{t h} C_{v} \nabla \bar{T}\right)-\nabla \cdot(\bar{p} \tilde{u})+\nabla \cdot Q_{S G S}
\end{gathered}
$$

where $\tau_{S G S}$ and $Q_{S G S}$ are the SGS stress and SGS heat flux resulting from the nonlinearity of the governing equations and thus need to be modeled.

In this work, $\tau_{S G S}$ is modeled as $\tau_{S G S}=-2 \mu_{S G S}\left(\tilde{S}_{i j}-\frac{\delta_{i j}}{3} \tilde{S}_{k k}\right)+$ $\frac{2}{3} \bar{\rho} k_{S G S} \delta_{i j}$, where the subgrid eddy viscosity $\mu_{S G S}$ and subgrid kinetic energy $k_{S G S}$ are modeled by the one-equation kinetic energy model.

$\frac{\partial \bar{\rho} k_{S G S}}{\partial t}+\nabla \cdot\left(\bar{\rho} \tilde{u}_{j} k_{S G S}\right)=\nabla \cdot\left[\left(\frac{\mu_{S G S}}{\operatorname{Pr}_{t}}+\mu\right) \nabla k_{S G S}\right]+P_{S G S}-\varepsilon_{S G S}$,

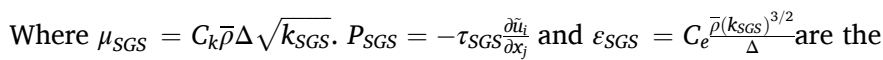
subgrid's production and dissipation of kinetic energy, respectively. $C_{k}=0.094$ and $C_{e}=1.048$ are constants.

The subgrid heat flux $Q_{S G S}$ is modeled as

$Q_{S G S}=-\frac{\mu_{S G S}}{\operatorname{Pr}_{t}} \nabla T$,

where $\operatorname{Pr}_{t}$ is the subgrid's turbulent Prandtl number that takes the value of 1.0 herein.

\subsection{Simulation procedure}

A slender cylindrical projectile with a $90^{\circ}$ conical head is used. The origin of the coordinates is defined at the center of the interface between the conical head and the projectile body. The $X O Y$ plane is parallel to the static free surface in space. The axis of the projectile is the $Z$-axis and is oriented to the conical head in the positive direction and perpendicular to the $X O Y$ plane. The diameter of the projectile is defined as $D$, the projectile length $L=10 D$, and the computing domain is a cylinder with a diameter of $12 \mathrm{D}$ and a length of $26 \mathrm{D}$, as shown in Fig. 2(a). The projectile is fixed, the liquid flows from the velocity inlet with a constant speed, and the pressure outlet is set to a standard atmospheric pressure. That means our simulations are based on projectile reference system instead of ground system. We do not use any kind of dynamic mesh strategy. Bilandi et al. (2018) also adopted similar method to simulate water entry process, its results show good agreement with previous works. A boundary condition called wavetransmissive is applied to eliminate possible wave reflections on outside boundaries.

A structured mesh is generated in the computational domain, the $y+$ in boundary layer is approximately equal to 1 , as shown in Fig. 2b The open source fluid mechanics software OpenFOAM ${ }^{\circledR}$ is employed to complete the calculation. The first-order implicit discretization is adopted in time discretization. Although second order discretization method is more accurate in theory, we still adopt first order scheme here, which due to the fact that it enables us to decrease the calculation time. Gauss linear interpolation is used in space. The time step is controlled by a Courant number and the maximum courant number is set to $\mathrm{Co}<0.5$.

We adopt grid convergency index (GCI) method to check mesh independency. The mesh we use are listed in Table 2. The GCI predicts discretization value relative to the value of the asymptotic numerical value, and it can reveal whether the results are within the range of convergence. According to Roache (1997), the GCI for fine grid can be defined as: 
(a)

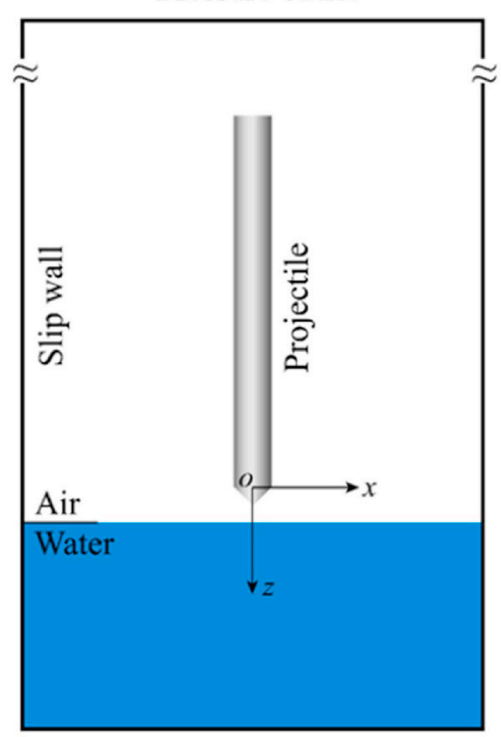

Velocity inlet (b)

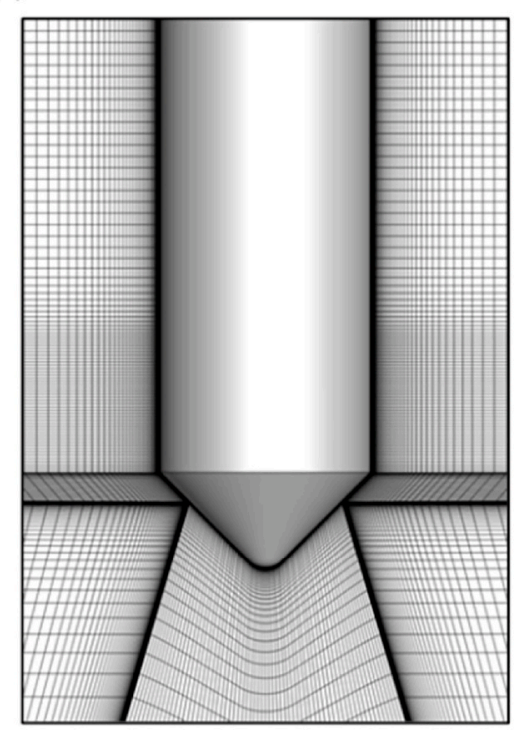

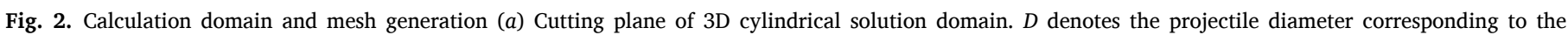
experimental specimen with a length of $9 \mathrm{~mm}$. (b) Mesh elements in the vicinity of the conical head of the test model.

Table 2

Grid convergence study for projectile water entry.

\begin{tabular}{llll}
\hline Number & Cells & Maximum $C d$ & CPU-hours \\
\hline 1 & 3930000 & 1.043345 & 400 \\
2 & 5895000 & 1.039974 & 1200 \\
3 & 8842000 & 1.033098 & 2600 \\
\hline
\end{tabular}

$G C I=\frac{F_{s}|\varepsilon|}{\left(\tau^{P}-1\right)}$,

where $F_{s}$ is a safe factor, the recommended value is $F_{s}=1.25$, and $\varepsilon$ is relative error. $\tau$ represents grid refinement ratio, here we have $\tau=1.5$. $\mathrm{P}$ indicates order of convergency, which could be obtained through $\tau$,

$P=\ln \left(\frac{f_{3}-f_{2}}{f_{2}-f_{1}}\right) / \ln (\tau)$,

$\mathrm{f}$ indicates the corresponding value of physical character. In our case, the theoretical value of $\mathrm{P}$ is 1.5, whereas the calculated number is 1.748 . Hence, The GCI for grid 1 and grid 2 is $0.391 \%$ while $0.793 \%$ for grid 2 and 3. Since $G C I_{2,3} / \tau^{p} G C I_{1,2} \approx 0.998$ which is approximately equal to 1 , we can conclude that the solutions are well within the asymptotic range of convergence. Since the results show only a slight difference in maximum drag coefficients for different mesh sizes, mesh 3 is safely chosen.

The model is validated by comparing the numerical and experimental results. The cavity shapes are extracted by iso-surface $\alpha_{l}=0.9$ when $F r=31.91$. The results indicate the agreement between the experiments and the simulation methods in terms of cavity shape (Fig. 3).

\section{Results and discussion}

\subsection{Drag coefficient}

The variation of drag force versus $t^{*}$ during the whole process of water entry is recorded in the case of $\mathrm{Fr}=31.91$ and nondimensional time $t *=t v_{0} / L, t^{*}=0$ is defined at the moment when the conical head vertex touches the free surface (Fig. 4A). The drag force suddenly
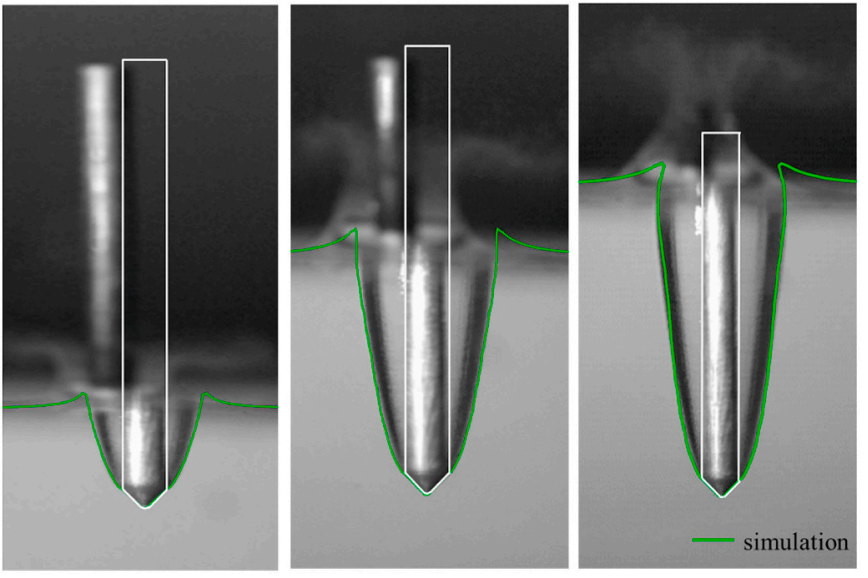

Fig. 3. Comparison of experimental and simulated cavity profiles (green line) for a projectile water entry with $F r=31.91$. The images of the test model are distorted due to light reflection at the cavity interface. Thus, the actual projectile profiles are included (white line).

changes and reaches its peak value when a large proportion of conical head is submerged underwater in a short time as shown in Fig. 4(B). Subsequently, the conical head is fully submerged, and the drag force drops rapidly, thus achieving a stable value. Fig. 4 shows the liquid fraction distribution at different $t^{*}$ obtained in the simulation. The formation and closure of the crown-shaped water splash, as well as the droplet scattering, show the same trend as the experimental results.

The drag coefficient varying with $t *$ is measured, and the numerical results with the different initial entry velocities of 30, 35, 40, 45, and 50 $\mathrm{m} / \mathrm{s}$ are repeated. The corresponding $\mathrm{Fr}$ numbers are 31.91, 37.23, 42.55, 47.87, and 53.19. The time sequences of drag coefficients of different $\mathrm{Fr}$ numbers are shown in Fig. 5, the resistance are nondimensionalized by $0.5 \rho v_{0}^{2} A$. The drag coefficients are generally independent of the Fr number under the aforementioned conditions.

The drag coefficient in the process of water entry is compared with those in underwater navigation and supercavitation situations (as shown in Fig. 6). The results of the immersed and supercavitation conditions 
(a)

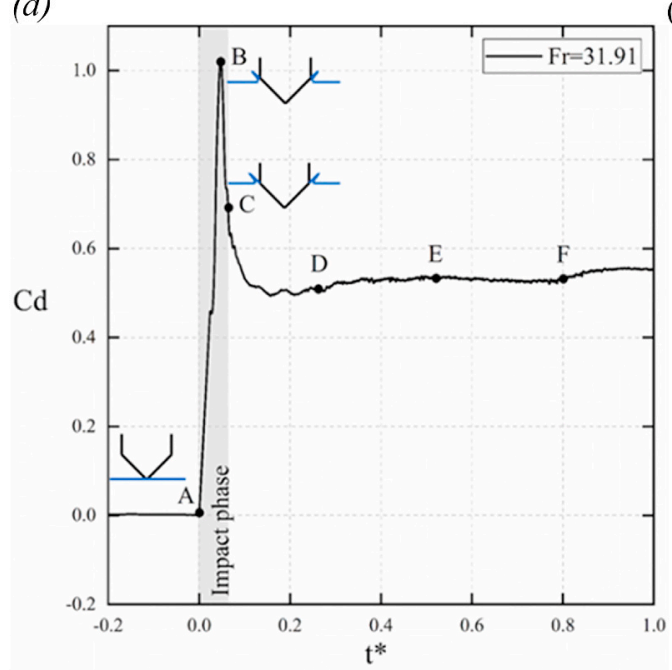

(b)

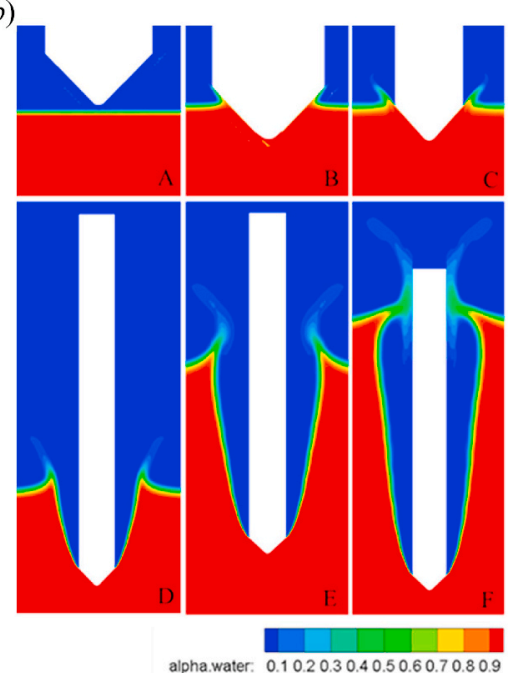

Fig. 4. (a) Evolution of drag coefficient from water entry of projectile with $F r=31.91$. (b) Cavity profiles corresponding to the letters $A-F$ marked in (a), and $t^{*}=0$, $\mathrm{t}^{*}=0.048, \mathrm{t}^{*}=0.063, \mathrm{t}^{*}=0.26, \mathrm{t}^{*}=0.53, \mathrm{t}^{*}=0.80$ respectively. The drag coefficient reaches its peak value when around half of the conical head is submerged in water.
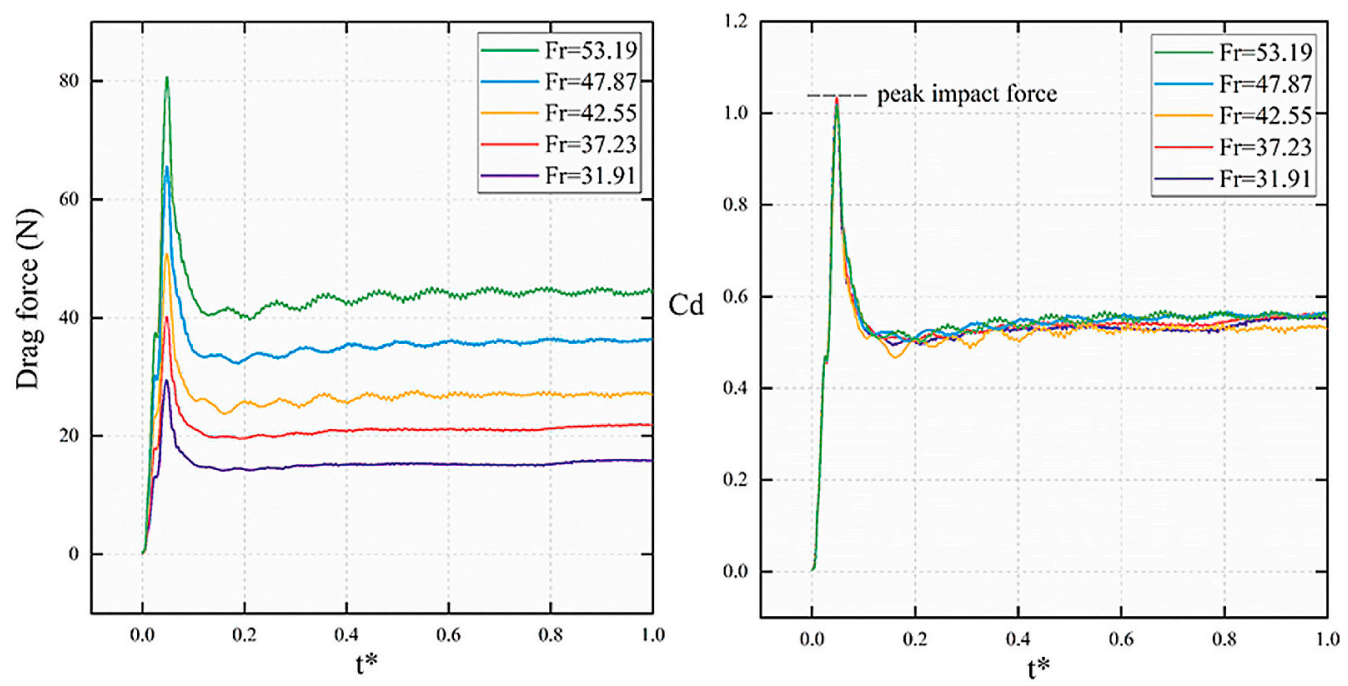

Fig. 5. Drag force and nondimensional drag force of projectile during water entry.

(a)
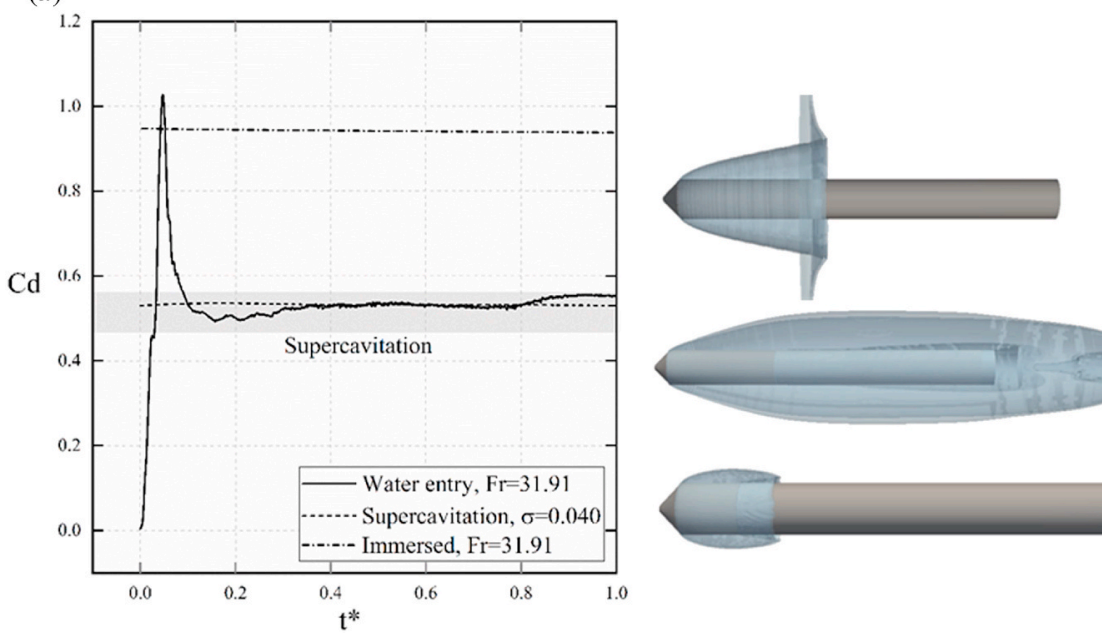
$30 \mathrm{~m} / \mathrm{s}$ ). In the supercavitation case, the incoming flow velocity is $70 \mathrm{~m} / \mathrm{s}$, which corresponds to the cavitation number $\sigma=0.040$. The drag coefficients under supercavitation and immersion are obtained from a steady state. The measured data for the supercavitation case fluctuates within the gray region for a long period. Schematic shows the corresponding cavity profiles of water entry, supercavitation, and submerged navigation $(b)-(d)$. 
are obtained by using the same computational grid and solver. We find that the drag coefficient under full immersion is much greater than that in the stable stage during water entry. By contrast, the drag coefficient under supercavitation is close to that during water entry when $C d$ becomes quasi-constant. During the procedure of water entry, the projectile is wrapped in an air-entering cavity, and only a small part of the projectile nose comes into contact with the water. Therefore, the viscous drag is neglectable and the drag coefficient is much smaller than that in the submersion state of underwater navigation. Although the internal mechanism of water entry is completely different from that under supercavitation, their effects of drag reduction are similar. Vincent et al. (2018) studied experimentally in detail the drag coefficient history during wedge water entry at different speeds with $F_{r} \in[1.7,5.0]$ and suggested that the drag coefficient for water entry is about half of that for underwater navigation. Hence, drag prediction of water entry with the maturational theory of supercavitation is feasible.

\subsection{Conical head pressure distribution}

Fig. 7 shows the pressure $P$ evolution on the location of the conical head vertex with dimensionless time $t^{*}$ for a series of $F r$ numbers. The entry velocity is prescribed. It is similar to the change of the drag force that causes the pressure to reach its peak in a short period after contacting the quiescent water surface and then decrease rapidly to reach a quasi-constant value. When we increase the initial water entry velocity, the pressure experienced by the conical head of the projectile rises correspondingly. On the basis of this condition, the relationship between the pressure coefficient $\mathrm{Cp}$ and dimensionless time is given, with the pressure coefficient defined as $C p=P /\left(0.5 \rho v_{0}^{2}\right)$. The peak value of the pressure coefficient $C p$ in the early stage of water entry increases with the Fr number, whereas it is almost independent of the Fr after the initial impact stage. The maximum pressure coefficient increases with the $\mathrm{Fr}$ number. Hence, the pressure distribution is affected by Fr, that is, a high Fr causes the pressure to become concentrated on the vertex.

When a conical head projectile enters the water, only a small part contact with the liquid. Hence, the hydrodynamic loads are concentrated within a limited area. Therefore, the pressure may cause structural deformation and instability. Fig. 8 shows the pressure distribution near the conical head. The pressure gradient changes dramatically, and the pressure peaks at the apex of the conical head. At the shoulder of the body, the flow passes through and separates from the projectile body, and the pressure in this area reaches the lowest value, that is, below the ambient pressure.

Fig. 9 plots the pressure distribution on the projectile along the z-axis

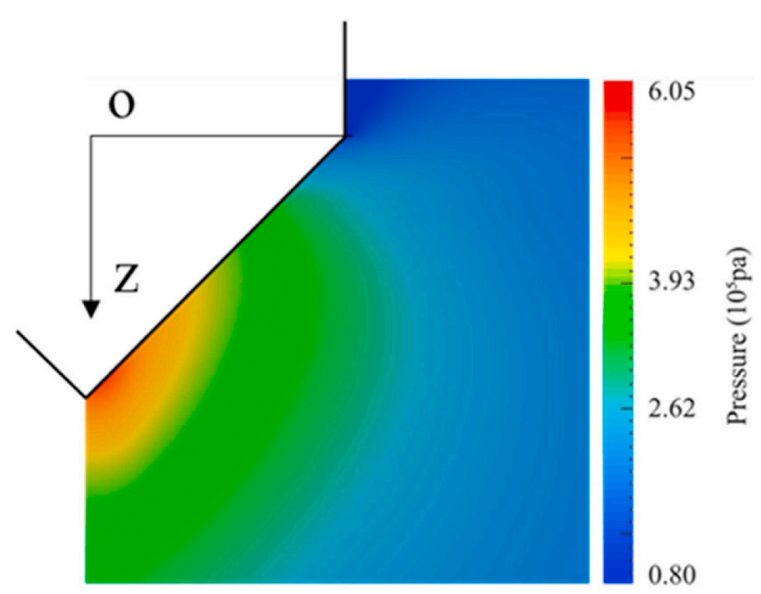

Fig. 8. Pressure contours near the conical head during the water entry of the projectile with constant velocity at $\mathrm{t}^{*}=0.267$.

at different $t^{*}$. When $t^{*}=0.067$ in the early stage of water entry, the pressure around $z=0.001$ is significantly higher than that in other moments. This trend of pressure distribution similar with $t^{*}=0.133$. The pressure decreases gradually from the apex to the bottom of the cone head cone with the increase in dimensionless time.

We find that pressure increases with the $F r$ number. We then compare the pressure coefficient distributions along the $\mathrm{z}$ axis for a range of Fr numbers. The results show that the pressure coefficient on the head of the projectile is independent of the Fr number, as shown in Fig. 10.

\subsection{Cavity profiles}

Cavity shape has always been an important issue. In this work, we mainly focus on cavity profile evolution that varies with different $t^{*}$ and Fr numbers when surface seal occurs prior to deep seal.

Fig. 11(a) illustrates the cavity profile evolution during the process of projectile water entry with $t^{*}$. We transform the horizontal free surface to the same height by defining $z^{\prime}=z+v_{0} t$. The profiles presented by the iso-surface of $\alpha_{l}=0.9$. The size of the cavity increases with $t^{*}$. Within the scope of our study, the temporal variations of cavity volume is shown in Fig. 11(b), the diameter of the cavity radius at original free surface section increases rapidly at first due to the pressure difference between the inside and outside parts of the cavity. The expansion speed slows down continuously and begins to shrink until surface closure occurs. (a)

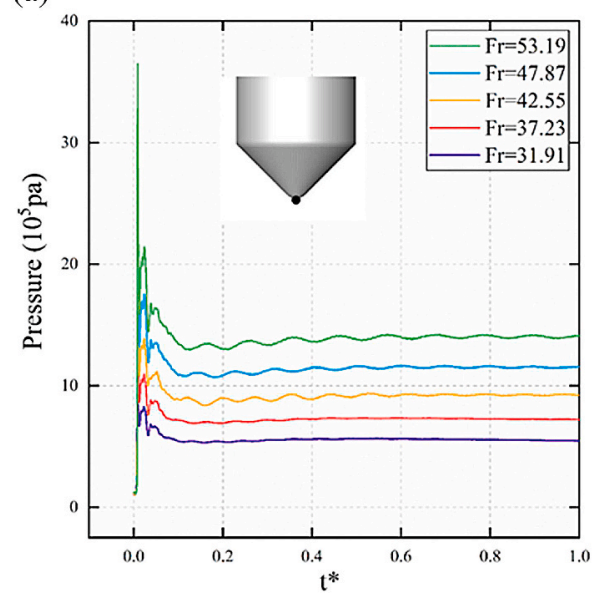

(b)

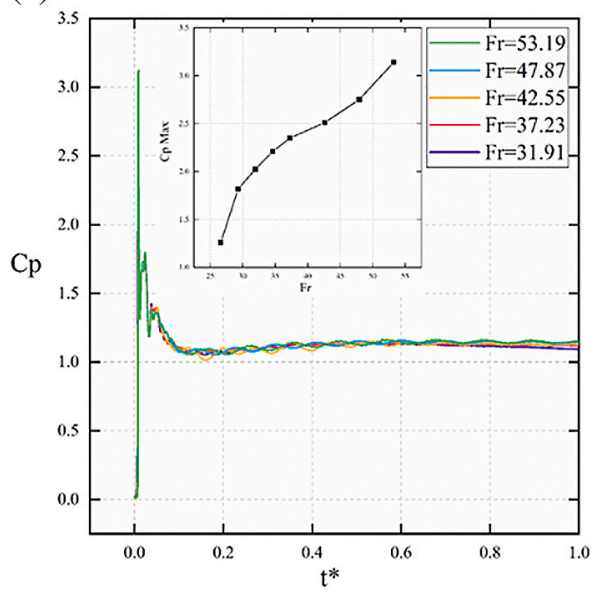

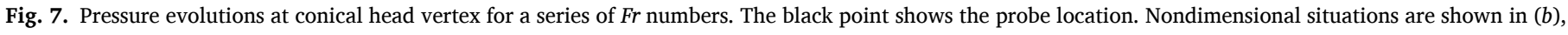
along with the maximum values of $\mathrm{Cp}$ for different $\mathrm{Fr}$ numbers. 


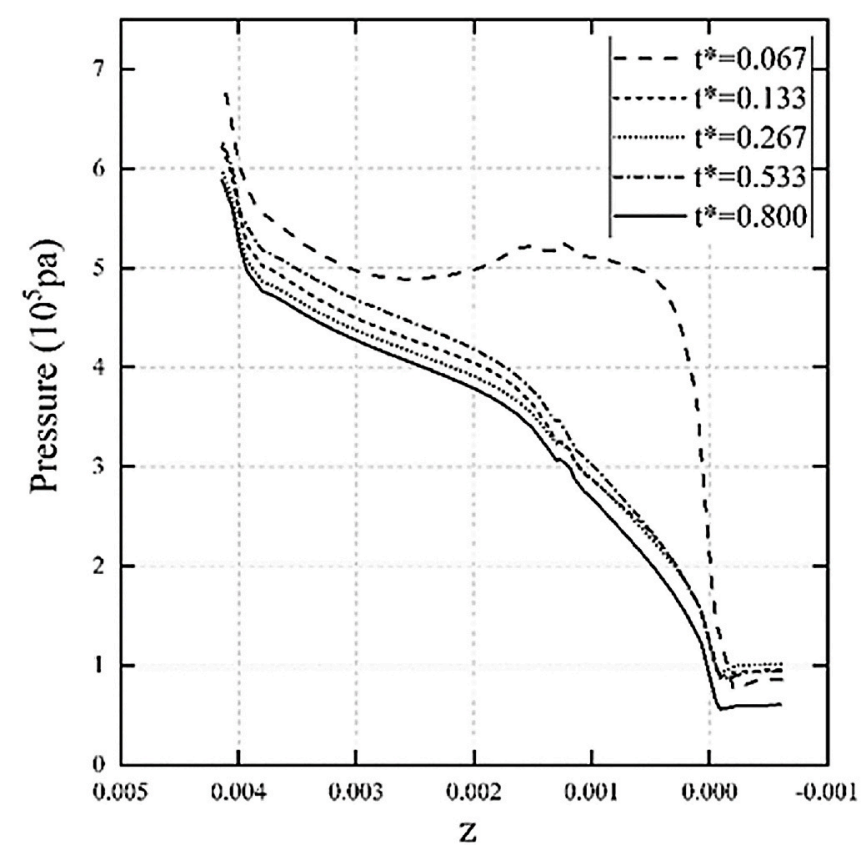

Fig. 9. Pressure distributions on the conical head alongside the z-axis at different nondimensional times.

Fig. 12 shows the instantaneous cavity profiles at different $t$ * for a range of Fr numbers. The profiles presented by the iso-surface of $\alpha_{l}=$ 0.9. In the early stage of water entry, the cavity profiles are basically coincidental. The influence of the $F r$ number gradually becomes apparent at $t^{*}=0.800$. The differences in cavity shapes become notable. The larger the Fr number is, the more the free surface will shrink inward. The results obtained by Kiara et al. (2017) also indicated that the effects of the Fr number on cavity profiles appear gradually during a cylinder's water entry. To reveal the mechanism behind this phenomenon, we introduce the prediction equations of the cavity radius proposed by Lee et al. (1997). The radius of cavitation can be expressed as follows:

$a\left(z_{b}, t\right)-a_{0}=\sqrt{[A(z)]^{2}-\left[A(z)-B(z)\left(t-t_{b}\right)\right]^{2}}$,

where

$[A(z)]^{2}=\frac{1}{\pi P_{g}} m \frac{\rho_{l} A_{0} C d}{2 m} v_{0}^{2}$,

$[B(z)]^{2}=\frac{P_{g}}{\rho_{l} N}$,

where $P g$ is the pressure difference across the air-liquid interface, $N$ is a geometric parameter with constant value, the letter $a$ represents cavity radius, $Z_{\mathrm{b}}$ indicates location of the projectile vertex and $m$ is projectile mass.

According to the previous calculation, the cavitation effect can be neglected under the conditions considered in this work. The equation can be simplified as follows:

$\left[a\left(z_{b}, t\right)-a_{0}\right]^{2}=2\left(t-t_{b}\right) \sqrt{\frac{A_{0} C d v_{0}^{2}}{2 \pi N}}-\frac{P_{g}}{\rho_{l} N} t^{2}$.

In consideration of $A_{0}=a_{0}^{2} \pi$, the equation can be written as

$\left[a\left(z_{b}, t\right)-a_{0}\right]^{2}=2 a_{0} V_{0} t \sqrt{\frac{C d}{2 N}}-\frac{P_{g}}{\rho_{l} N} t^{2}$.

The main factors that determine the cavity profiles are drag coefficient, water entry velocity, and pressure difference. Drag coefficient is highly related to nose shape and material properties. Cavity expansion is associated with drag coefficient and entry velocity while cavity closure is governed by pressure difference.

The cavity expands in the early stage of water entry. $P_{g} t^{2} / \rho_{l} N$ is much smaller than the first term on the right side of equal sign, so we neglect it. According to the previous definition of dimensionless time $t V_{0}=t^{*} L$, the radius of the cavity is retained in the same dimensionless time.

$\left[a\left(z_{b}, t\right)-a_{0}\right]^{2}=2 a_{0} t^{*} L \sqrt{\frac{C d}{2 N}}$

The faster the initial velocity is, the smaller the effect of the contraction term and the larger the size of the cavity will be when the object reaches the same depth. This result is consistent with the conclusion of the numerical simulation. Therefore, the time effect caused by the different initial velocities of water entry results in differences in cavity morphology.

According to Aristoff and Bush (2009), the pressure drops across the air-water interface involves three aspects, namely, the hydrostatic pressure affected by depth, the aerodynamic pressure caused by air entrainment, and the surface tension relevant to the free surface curvature. Hence, the pressure drops can be written as

$P_{g}=\sigma(\nabla \cdot \widehat{\boldsymbol{n}})+C_{a} \rho_{a} v_{a}^{2}+\rho g z$

where $C_{a}$ is a constant, $C_{a} \in[7.5,10], v_{a}$ is air flow speed. By substituting (4.3.7) into (4.3.5), we find that the lengths are scaled by $L$ and that the cavity's dynamic expressions can be rewritten in nondimensional forms as where $m$ ' indicates air-water mass ratio.

$\left[\frac{a\left(z_{b}, t\right)-a_{0}}{L}\right]^{2}=2 \frac{a_{0} t *}{L} \sqrt{\frac{C d}{2 N}}-\frac{1}{N}\left[\frac{B o}{W e} \cdot z *+\frac{\nabla \cdot \widehat{\boldsymbol{n}}}{W e} \cdot L+C_{a} m\left(\frac{v_{a}}{v_{0}}\right)^{2}\right] \times t *^{2}$

The expansion of the cavity radii is mainly determined by the drag coefficient and initial speed when $t^{*}$ is small at the early stage. The velocity can be manifested through nondimensional $\mathrm{Fr}$, because $t^{*}$ contains a velocity term. The cavity closure is affected by We and Bo. The dominant factors that determine cavity closure in different scales vary. Thus, different cavity patterns are present.

Note that the ratio of $B o$ to $W e$ can be rewritten as $1 / \mathrm{Fr}^{2}$ according to their definitions, in present work, since We and Fr are considerably large number, the influence of surface tension and hydrodynamic pressure can be neglected, thus the pressure drop caused by air entertainment is the key issue for surface seal, it also can be infer that the speed air flow reaches its maximum value at the cavity entrance, so cavity tends to seal at free surface due to Bernoulli effect. Aristoff and Bush (2009) also revealed that when $B o \cdot z * \gg 1$ and $F^{2} \ll m^{\prime}$, the hydrostatic pressure dominates cavity collapse, and the cavity pinch-off occurs at a certain depth. When surface tension occurs while hydrostatic pressure and aerodynamic are negligible, a shallow seal occurs.

For high Fr cases, the air flow properties, especially the air at the entrance is so important to determine cavity dynamics, so we further discuss the air flow behaviors inside the water entry cavity.

\subsection{Flow behavior inside the cavity}

The air cavity induced by water entry expands rapidly, and the interface reverses its direction before collapsing under the influence of pressure difference. This pressure difference is related to not only liquid properties, hydrodynamic pressure, and surface tension, but also aerodynamic pressure. Therefore, we examine the internal flow of the air cavity to determine the principle of aerodynamic pressure variation.

We notice that water flows smoothly around the projectile because the cavity induced by the water entry wraps around the projectile. In the air domain, a vertex ring emerges above the water surface, and then gas intrudes into the cavity under the influence of the vortices. The relative position between the vortices and the cavity, which gradually encapsulates the vortices, also changes with $t^{*}$. The portion of the vortex 
(a) $t^{*}=0.533$
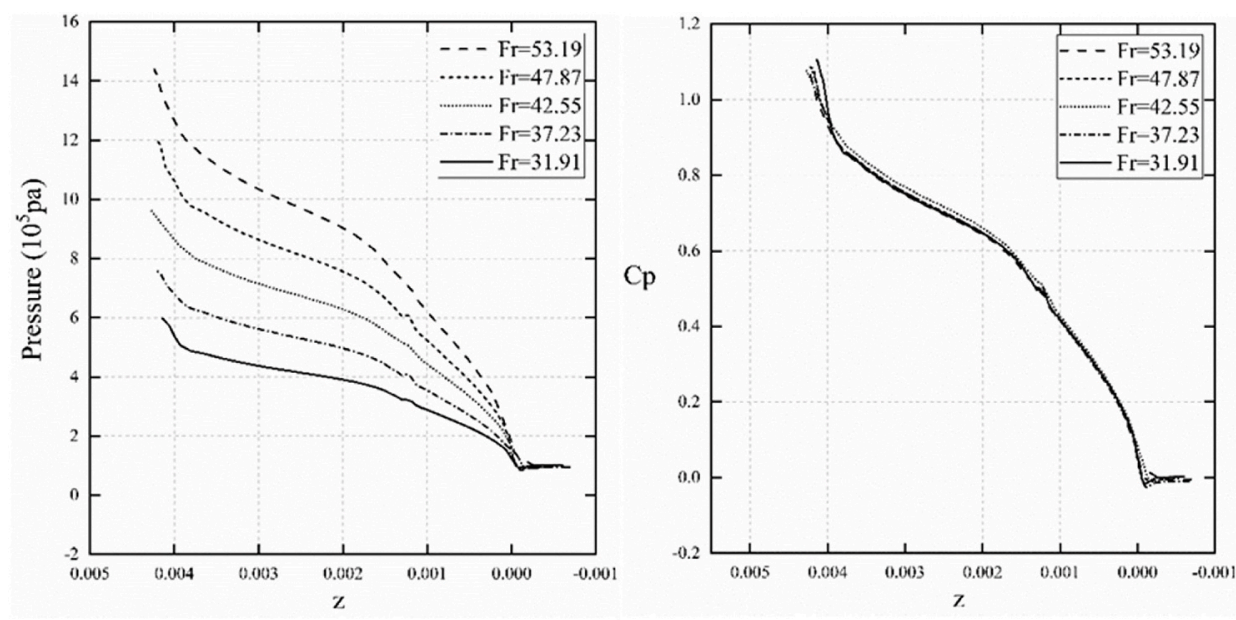

(b) $t^{*}=0.800$
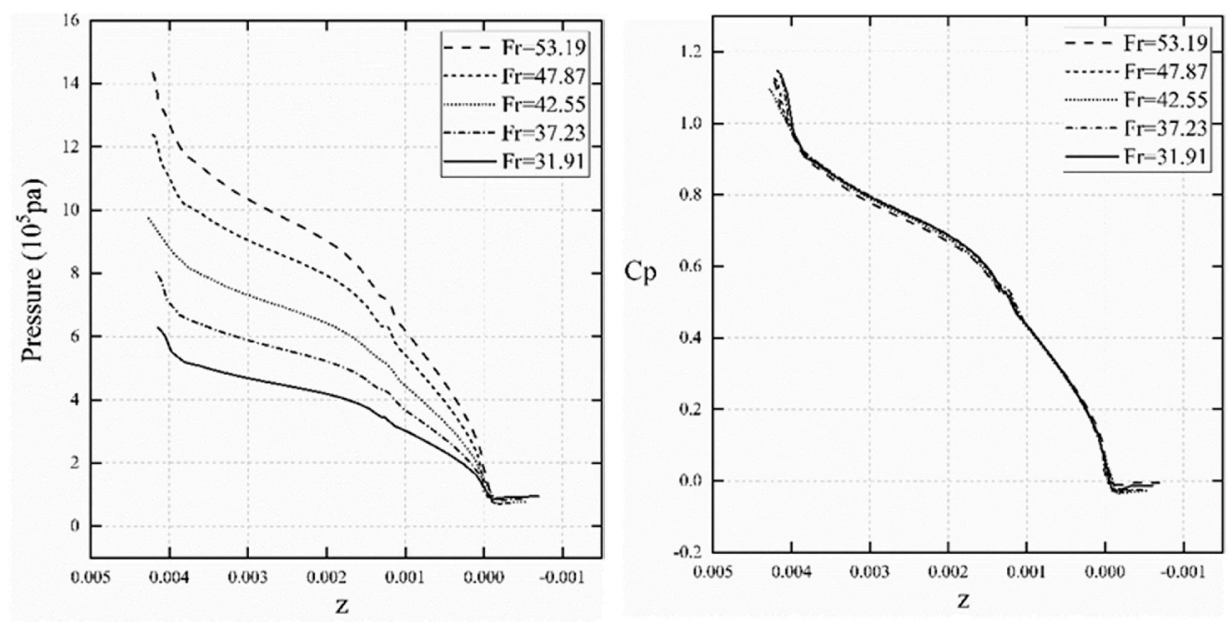

Fig. 10. Dimensional (left) and dimensionless (right) pressure distributions alongside the z-axis at (a) $t^{*}=0.533$ and (b) $t^{*}=0.800$ for different $F r$ numbers.
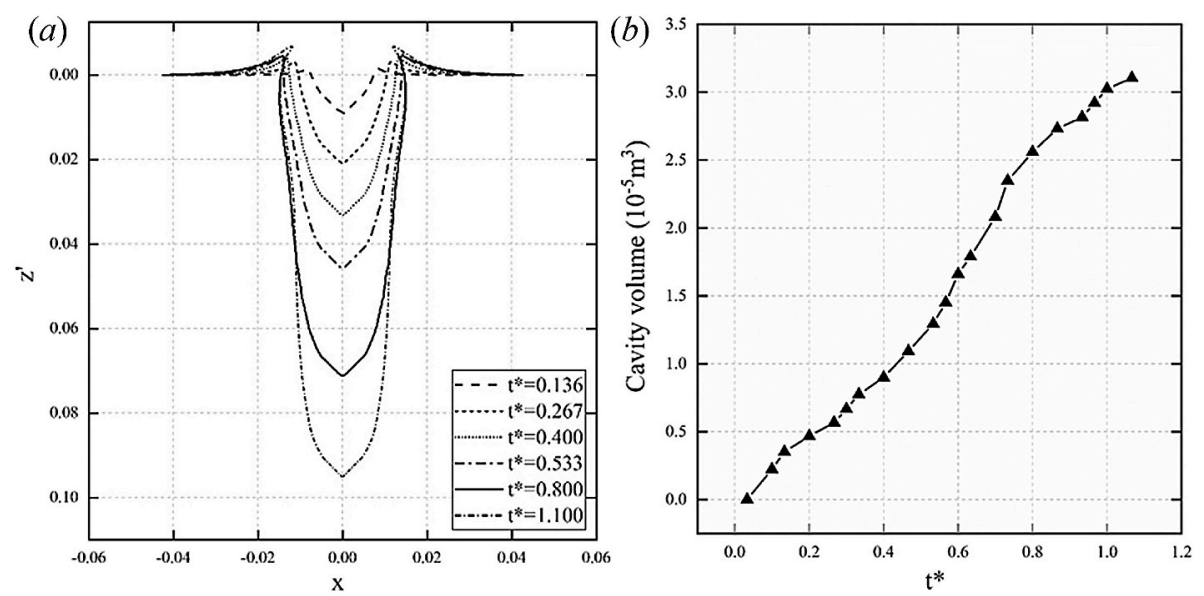

Fig. 11. Evolutions of cavity profiles and volume. (a) Cavity profiles of surface seal from a projectile water entry with a constant velocity at different nondimensional time periods. The cavity volume gradually increases due to projectile motion, although the cavity begins to contract at a certain moment (b).

contacting the air decreases continuously and tends to dissipate with the streamline changing from stable to chaotic (Fig. 13). The cavity shapes and vortices during the entry of air are closely related.
The velocity of the air inside the cavity can be observed clearly on the streamline (Fig. 14). In this work, we focus on the velocity distribution in the vorticity region. In the early stage of water entry, the maximum 
(a)

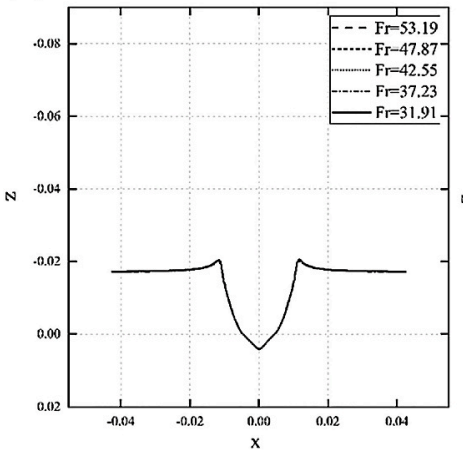

(b)

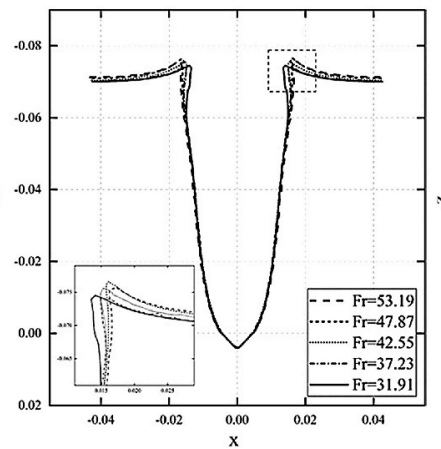

(c)

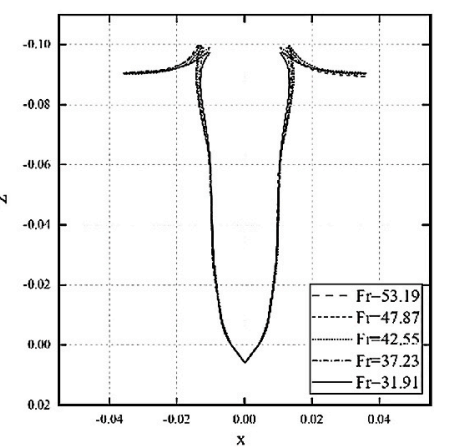

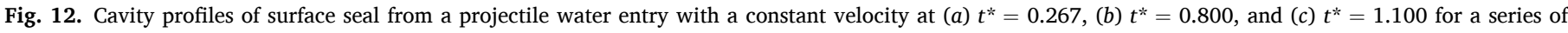
Fr numbers.

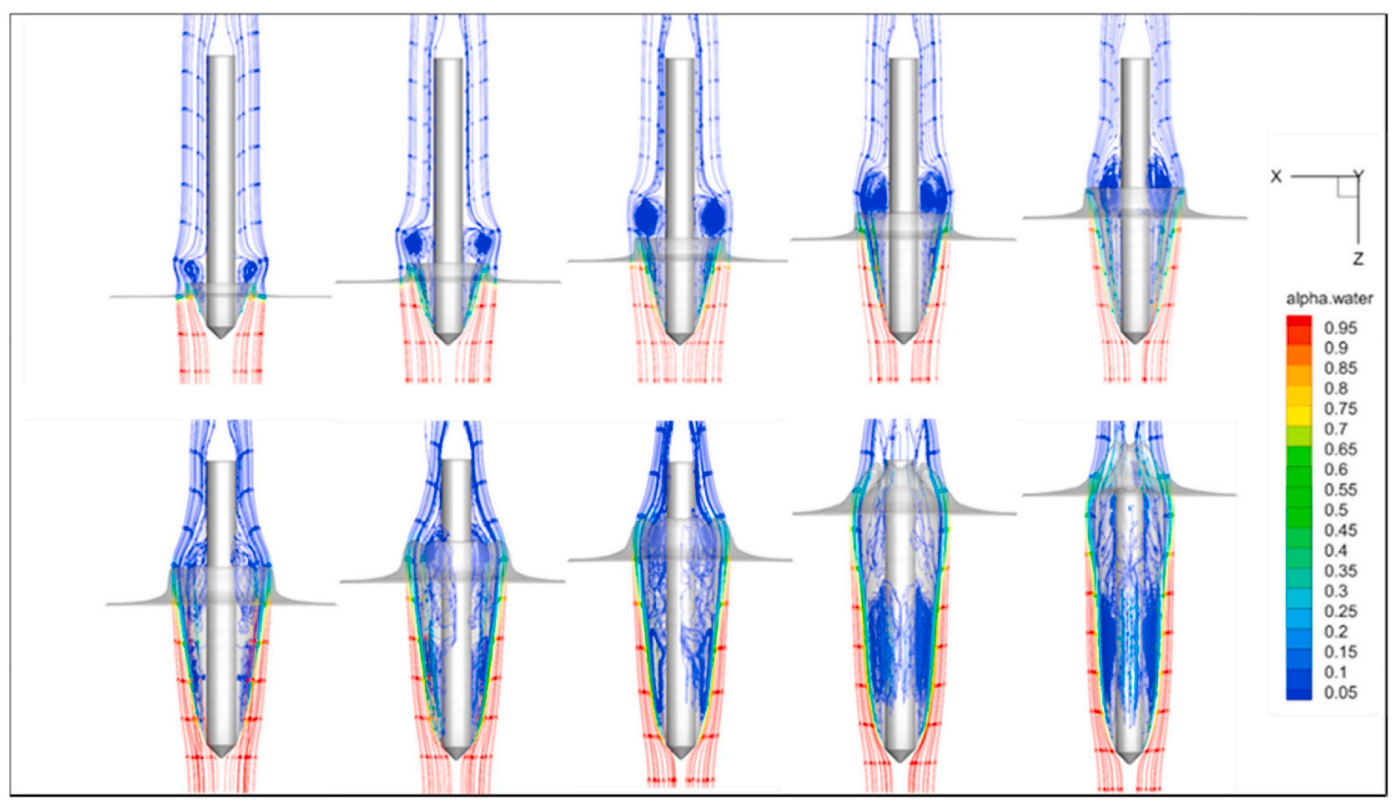

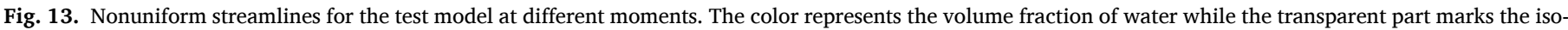
surface of the water fraction $\alpha_{l}=0.85$ indicating the cavity profiles. The coordinate is fixed on the projectile.
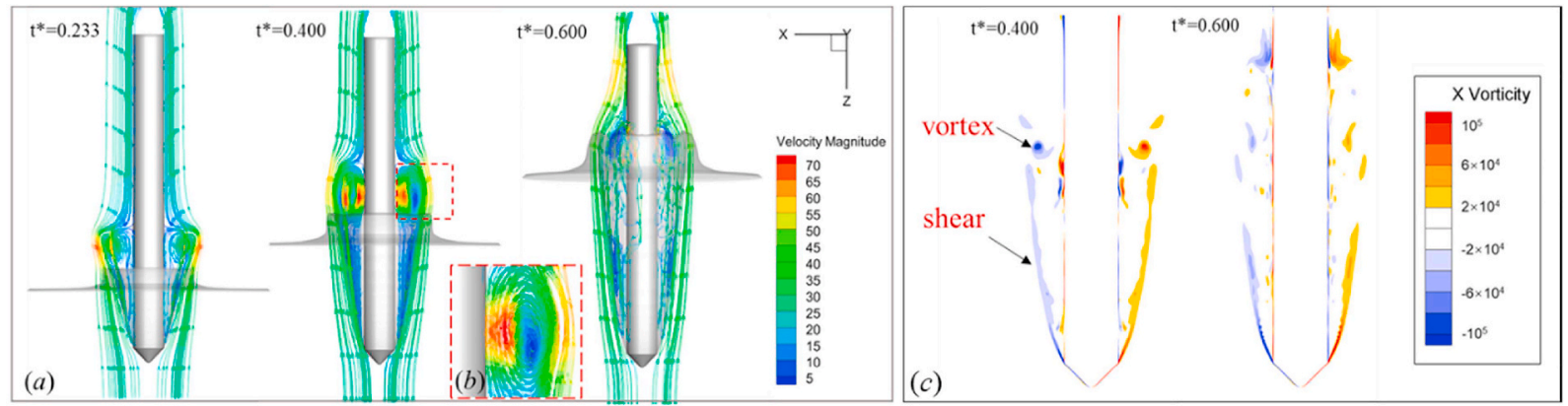

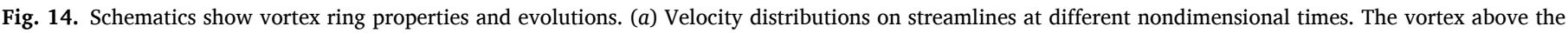

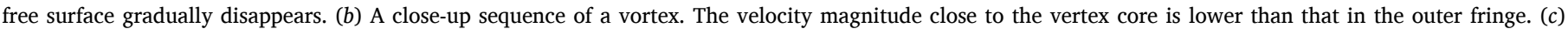

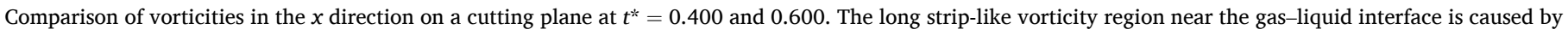
shear forces.

velocity in the flow field appears near the vortex ring and is faster than the air motion speed in the cavity. The velocity at the outer edge of the vortex can be affected when the air contacts the projectile wall. Within a certain range, the farther away air is from the vortex core, the faster the air rotates at $t^{*}=0.400$. In addition, the vortex almost dissipates at $t^{*}=$ 0.600 , in which case the velocity difference of the flow field in the cavity is minimal.

The pressure in the vicinity of the vortices at the entrance of the 
horizontal water surface is obviously lower than the cavity pressure and air domain. Thus, surface seal precedes deep seal due to the pressure difference at the cavity entrance (Fig. 15). We also note that the pressure at the entrance forms a circular contour with a distinct pressure gradient. The relative position between the low-pressure region and the free surface changes with time, moving from the outside of the cavity to the inside. One can easily infer according to a previous work (e.g. Jeong and Hussain, 1995) that the generation of this low-pressure region is related to the vortex motion. In the shoulder of the object, a low-pressure zone with a considerable change in pressure gradient is formed due to flow separation. The figure shows pressure distribution at $t^{*}=0.267$, the free water surface is close to the closure, and the vortices dissipate. Therefore, the low-pressure zone near the cavity entrance disappears.

Except for the obvious pressure drop at the cavity entrance and the shoulder of the projectile, the pressure changes in the cavity are relatively smooth (as shown in Fig. 16). The average pressure in the cavity decreases with time from $t^{*}=0.267$. This result is due to the motion of the splash and the gradual dissipation of the vortices, both of which prevent the air from intruding into the cavity. Meanwhile, the projectile moves underwater, and the volume of the cavity increases. According to the isentropic relation, the air density and cavity pressure decrease. By contrast, in the initial stage of water entry, the cavity is open and connected with the atmosphere, and the air flow can enter the cavity through the vortices. Therefore, the relative stability of the density pressure in the cavity is maintained. The vortex motion and its chain effect eventually lead to the closure of the cavity. Besides, the lowest pressure in the cavity is always higher than the saturated vapor pressure in the scope of our studies $(F r=30.91-53.19)$; therefore, no cavitation occurs. In other words, no vapor is generated by the phase change in the cavity. A possible scenario is ambient air entrances into the cavity, which increases the inner pressure. However, the pressure in the cavity tends to decrease with the dimensionless time $t^{*}$ and Fr number. Thus, when the Fr number increases further, cavitation may occur. However, the pressure in the cavity tends to decrease with the dimensionless time $t^{*}$ and Fr number. Thus, when the Fr number increases further, cavitation may occur.

\section{Conclusion}

We study the water entry problem of a 3D slender conical head projectile with a prescribed velocity in the case of a surface seal occurring prior to deep seal. Our goal is to numerically investigate the

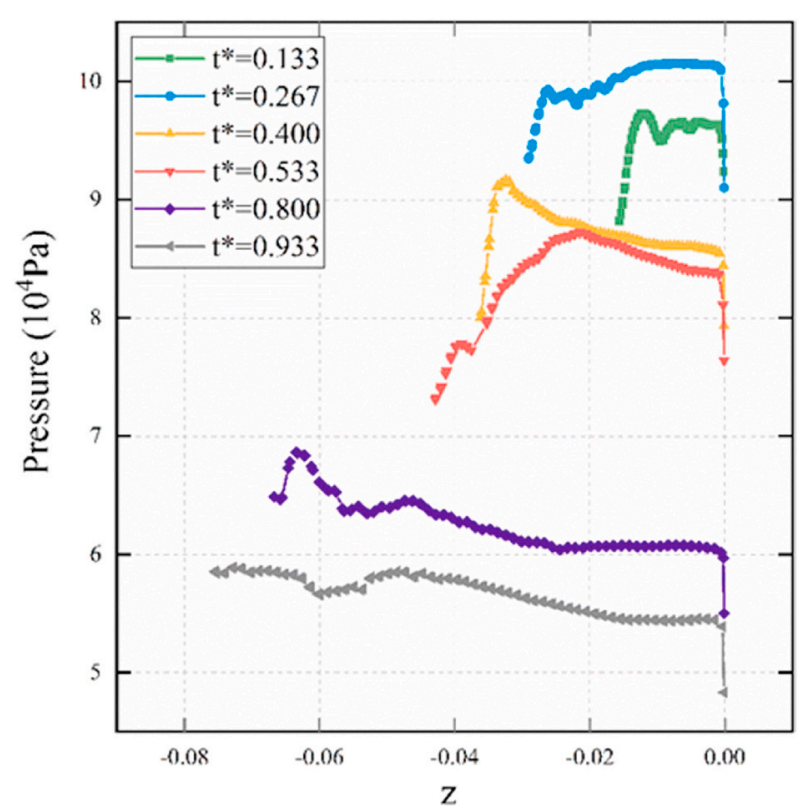

Fig. 16. Schematic showing the variations of internal pressure along the generatrix of projectile body at different dimensionless time periods with $F r=31.91$ on the symmetric plane. Note that $\mathrm{z}=0$ is the location of projectile shoulder.

flow characteristics during water entry process. The VOF method is employed to capture free surface motion. LES is used to simulate turbulence. A pressure-based compressible multiphase solver with cavitation phase change is developed in the framework of the OpenFOAM $₫$ source code. The simulation results are proven to be credible following a comparison with the experiment outcomes.

We first discuss the slamming load act on the projectile when the surface closure takes precedence, results show the drag coefficient, which is independent of the Fr number in general, is similar to that in the case of supercavitation and is lower than that in underwater navigation. We also find that the pressure at the vertex of the projectile also increases dramatically in a short time before stabilizing but the maximum pressure coefficient $C p$ on the vertex increases with the Fr number.

Subsequently, the effects of initial velocity on cavity dynamics are discussed. We showed that In the early stage of water entry, the profile of the cavity is basically independent of $F r$, as t* passes, differences in
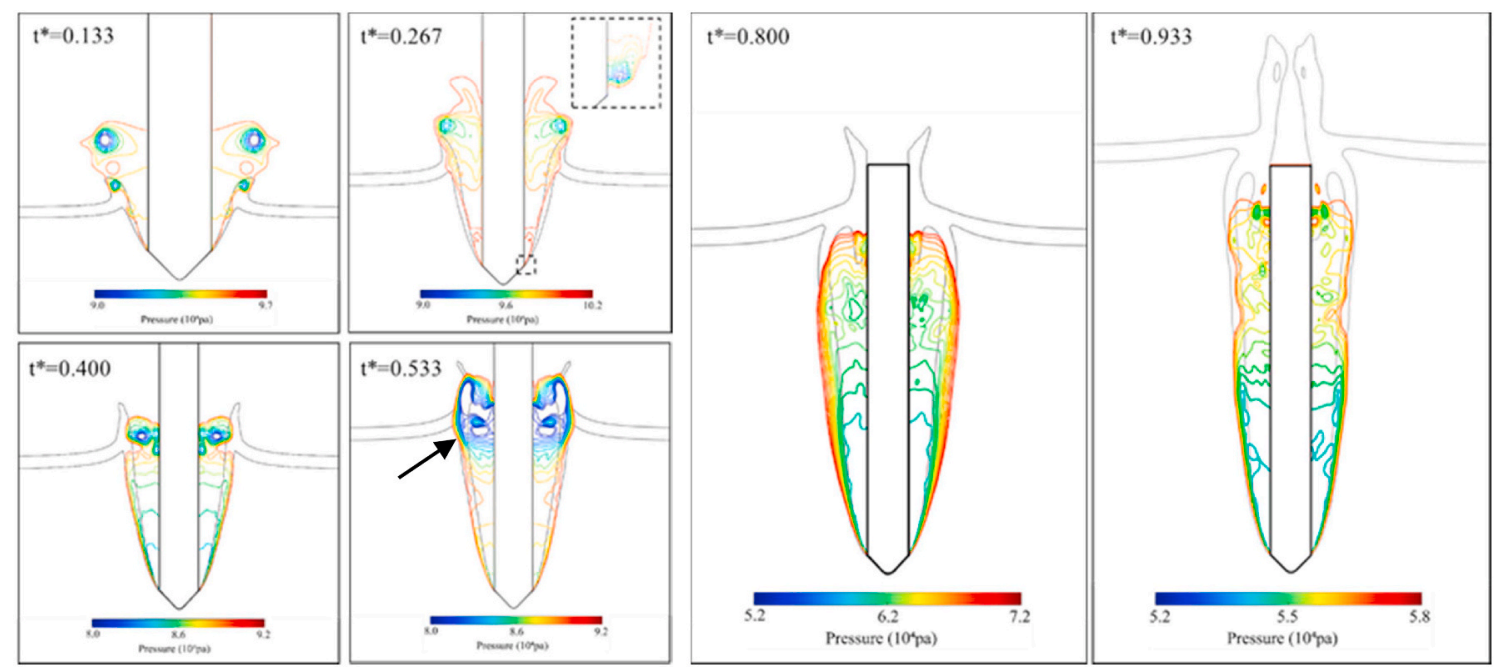

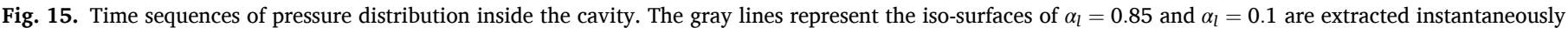
which can be considered to be cavity boundary. The black arrow shows the low pressure region induced by vortex ring. 
cavity shapes gradually appear due to the different initial entry velocities, airflow can also play an important role in cavity shapes when $F r$ is high enough.

Then we further explore the physics of gas flow inside the bubble. Vortex rings are observed near the horizontal free surface. The pressure near the position of the vortex ring is obviously lower than that in the remaining fields inside cavity, the existence of vortex ring make contributions to cavity sealing.

We find that the flow characteristics inside the cavity play an important role in free surface evolution. Therefore, cavity formation is determined by the interaction between air and water. In the future, more in-depth work, for example the flow visualizations inside the cavity should be carried out.

\section{CRediT authorship contribution statement}

Yufei Wang: Investigation, Methodology, Formal analysis, Validation, Resources, Writing - original draft. Bingsheng Ye: Data curation, Validation, Software. Zhiying Wang: Visualization, Writing - review \& editing. Jian Huang: Validation. Yiwei Wang: Project administration, Writing - review \& editing, Supervision, Conceptualization. Chenguang Huang: Funding acquisition.

\section{Declaration of competing interest}

The authors declare that they have no known competing financial interests or personal relationships that could have appeared to influence the work reported in this paper.

\section{Acknowledgement}

Project supported by the National Key R\&D Program (Grant No. 2016YFC0300800, 2016YFC0300802. The authors would also like to thank the National Natural Science Foundation of China through Grant No. 11772340, 11802311 and 11672315, and the Youth Innovation Promotion Association CAS (201906).

\section{References}

Abelson, H., 1970. Pressure measurements in the water-entry cavity. J. Fluid Mech. 44 129-144.

Aristoff, J.M., Bush, J.W.M., 2009. Water entry of small hydrophobic spheres. J. Fluid Mech. 619, 45-78.

Battistin, D., Iafrati, A., 2003. Hydrodynamic loads during water entry of twodimensional and axisymmetric bodies. J. Fluid Struct. 17 (5), 643-664.

Bilandi, R.N., Jamei, S., Roshan, F., Azizi, M., 2018. Numerical simulation of vertical water impact of asymmetric wedges by using a finite volume method combined with a volume-of-fluid technique. Ocean. Eng. 160, 119-131.

Bodily, K.G., Carlson, S.J., Truscott, T.T., 2014. The water entry of slender axisymmetric bodies. Phys. Fluids 26 (7), 072108.

Calderer, A., Kang, S., Sotiropoulos, F., 2014. Level set immersed boundary method for coupled simulation of air/water interaction with complex floating structures. J. Comput. Phys. 277, 201-227.

Chang, B., Croson, M., Straker, L., Gart, S., Dove, C., Gerwin, J., Jung, S., 2016. How seabirds plunge-dive without injuries. Proc. Natl. Acad. Sci. U. S. A. 113 (43), 12006-12011.

Clanet, C., Hersen, F., Bocquet, L., 2004. Secrets of successful stone-skipping. Nature 427 (6969), 29-29.

Dobrovol'skaya, N., Z, 1969. On some problems of similarity flow of fluid with a free surface. J. Fluid Mech. 36, 805-829.

Duclaux, V., Caille, F., Duez, C., Ybert, C., Bocquet, L., Clanet, C., 2007. Dynamics of transient cavities. J. Fluid Mech. 591, 1-19.

Elhimer, M., Jacques, N., Alaoui, A.E., Gabillet, C., 2017. The influence of aeration and compressibility on slamming loads during cone water entry. J. Fluid Struct. 70, 24-46.

Erfanian, M.R., Anbarsooz, M., Rahimi, N., Zare, M., Moghiman, M., 2015. Numerical and experimental investigation of a three dimensional spherical-nose projectile water entry problem. Ocean. Eng. 104, 397-404.

Gong, K., Shao, S.D., Liu, H., Lin, P.Z., Gui, Q.Q., 2019. Cylindrical smoothed particle hydrodynamics simulations of water entry. J. Fluid Eng.Trans. ASME 141 (7), 071303.

Hicks, P.D., Ermanyuk, E.V., Gavrilov, N.V., Purvis, R., 2012. Air trapping at impact of a rigid sphere onto a liquid. J. Fluid Mech. 695, 310-320.
Howison, S.D., Ockendon, J.R., Wilson, S.K., 1991. Incompressible water-entry problems at small deadrise angles. J. Fluid Mech. 222, 215-230.

Hsieh, S.T., Lauder, G.V., 2004. Running on water: three-dimensional force generation by basilisk lizards. Proc. Natl. Acad. Sci. U.S.A. 101 (48), 16784-16788.

Hu, D.L., Chan, B., Bush, J.W.M., 2003. The hydrodynamics of water strider locomotion. Nature 424 (6949), 663-666.

Jeong, J.J.J., Hussain, F., 1995. On the identification of a vortex. J. Fluid Mech. 332 (1), 339-363.

Johnson, W., 1998. The ricochet of spinning and non-spinning projectiles, mainly from water. Part II: an outline of the theory and warlike applications. Int. J. Impact Eng. $21,25-34$.

Kiara, A., Paredes, R., Yue, D.K.P., 2017. Numerical investigation of the water entry of cylinders without and with spin. J. Fluid Mech. 814, 131-164.

Kim, N., Park, H., 2019. Water entry of rounded cylindrical bodies with different aspect ratios and surface conditions. J. Fluid Mech. 863, 757-788.

Korobkin, A.A., Scolan, Y.M., 2006. Three-dimensional theory of water impact. Part 2. Linearized Wagner problem. J. Fluid Mech. 549, 343-373.

Kulkarni, S.S., Pratap, R., 2000. Studies on the dynamics of a supercavitating projectile. Appl. Math. Model. 24 (2), 113-129.

Kunz, R.F., Boger, D.A., Stinebring, D.R., Chyczewski, T.S., Lindau, J.W., Gibeling, H.J., Venkateswaran, S., Govindan, T.R., 2000. "A preconditioned Navier-Stokes method for two-phase flows with application to cavitation prediction. Comput. Fluids 29, 849-875.

Lee, M., Longoria, R.G., Wilson, D.E., 1997. Cavity dynamics in high-speed water entry. Phys. Fluids 9 (3), 540-550.

Louf, J.-F., Chang, B., Eshraghi, J., Mituniewicz, A., Vlachos, P.P., Jung, S., 2018. Cavity ripple dynamics after pinch-off. J. Fluid Mech. 850, 611-623.

Mansoor, M.M., Marston, J.O., Vakarelski, I.U., Thoroddsen, S.T., 2014. Water entry without surface seal: extended cavity formation. J. Fluid Mech. 743, 295-326.

Marston, J.O., Truscott, T.T., Speirs, N.B., Mansoor, M.M., Thoroddsen, S.T., 2016. Crown sealing and buckling instability during water entry of spheres. J. Fluid Mech. 794, 506-529.

May, A., 1953. Vertical entry of missiles into water. J. Appl. Phys. 23, 1362-1372.

May, A., 1975. Water Entry and the Cavity-Running Behavior of Missiles. Nasa Sti/recon Technical Report N.

Mcintyre, S, Kinzel, M, Miller, S, 2011. The immersed boundary method for water entry simulation. Aiaa Aerospace Sciences Meeting Including the New Horizons Forum \& Aerospace Exposition.

Mei, X.M., Liu, Y.M., Yue, D.K.P., 1999. On the water impact of general two-dimensional sections. Appl. Ocean Res. 21 (1), 1-15.

Nagahiro, S., Hayakawa, Y., 2005. Theoretical and numerical approach to "magic angle" of stone skipping. Phys. Rev. Lett. 94 (17), 174501.

Panciroli, R., Falcucci, G., Erme, G., De Santis, E., Jannelli, E., 2015. Fluid-structure interaction during the water entry of flexible cylinders. Proc. Int. Conf.Numer. Anal. Appl. Math. 2014 (Icnaam-2014) 1648.

Roache, P., 1997. Quantification of uncertainty in computational fluid dynamics. Annu. Rev. Fluid Mech. 29 (1), 123-160.

Seddon, C.M., Moatamedi, M., 2006. Review of water entry with applications to aerospace structures. Int. J. Impact Eng. 32 (7), 1045-1067.

Shams, A., Jalalisendi, M., Porfiri, M., 2015. Experiments on the water entry of asymmetric wedges using particle image velocimetry. Phys. Fluids 27 (2), 027103.

Speirs, N.B., Belden, J., Pan, Z., Holekamp, S., Badlissi, G., Jones, M., Truscott, T.T., 2019. The water entry of a sphere in a jet. J. Fluid Mech. 863, 956-968.

Sruthi, C., Sriram, V., 2017. Wave impact load on jacket structure in intermediate water depth. Ocean. Eng. 140, 183-194.

Sun, H., Faltinsen, O.M., 2006. Water impact of horizontal circular cylinders and cylindrical shells. Appl. Ocean Res. 28 (5), 299-311.

Tan, B.C.W., Vlaskamp, J.H.A., Denissenko, P., Thomas, P.J., 2016. Cavity formation in the wake of falling spheres submerging into a stratified two-layer system of immiscible liquids. J. Fluid Mech. 790, 33-56.

Truscott, T.T., Epps, B.P., Techet, A.H., 2012. Unsteady forces on spheres during freesurface water entry. J. Fluid Mech. 704, 173-210.

Truscott, T.T., Epps, B.P., Belden, J., 2014. Water entry of projectiles. Annu. Rev. Fluid Mech. 46 46, 355-378.

Vincent, L., Xiao, T., Yohann, D., Jung, S., Kanso, E., 2018. Dynamics of water entry. J. Fluid Mech. 846, 508-535.

Von Karman, T., 1929. The impact on seaplane floats during landing. NACA Tech. Rep. 321.

Wagner, H., 1932. Über stoß-und gleitvorgänge an der oberfläche von flüssigkeiten. Z. Angew. Math. Mech. 12, 193-215.

Wang, S., Soares, C.G., 2016. Experimental and numerical study of the slamming load on the bow of a chemical tanker in irregular waves. Ocean. Eng. 111, 369-383.

Wang, Y.W., Xu, C., Wu, X.C., Huang, C.G., Wu, X.Q., 2017. Ventilated cloud cavitating flow around a blunt body close to the free surface. Physical Review Fluids 2 (8), 084303.

Worthington, A.M., 1908. A Study of Splashes. Longmans, Green, and Co.

Xu, G.D., Duan, W.Y., Wu, G.X., 2008. Numerical simulation of oblique water entry of an asymmetrical wedge. Ocean. Eng. 35 (16), 1597-1603.

Xu, C., Huang, J., Wang, Y.W., Wu, X.C., Huang, C.G., Wu, X.Q., 2018. Supercavitating flow around high-speed underwater projectile near free surface induced by air entrainment. AIP Adv. 8 (3), 035016. 
Ye, B.S., Wang, Y.W., Huang, C.G., Huang, J., 2019. Numerical study of the pressure wave-induced shedding mechanism in the cavitating flow around an axisymmetric projectile via a compressible multiphase solver. Ocean. Eng. 187, 106179.
Zarghami, A., Falcucci, G., Jannelli, E., Succi, S., Porfiri, M., Ubertini, S., 2014. Lattice Boltzmann modeling of water entry problems. Int. J. Mod. Phys, C 25 (12), 1441012. Zhao, R., Faltinsen, O., 1993. Water entry of two-dimensional bodies. J. Fluid Mech. 246, 593-612. 